Article

\title{
Nuclear Physics at the Energy Frontier: Recent Heavy Ion Results from the Perspective of the Electron Ion Collider
}

\author{
Astrid Morreale ${ }^{1,2}$ (D) \\ 1 Institute for Globally Distributed Open Research, 75014 Paris, France; astrid.morreale@igdore.org or \\ astrid.morreale@gmail.com \\ 2 Center for Frontiers in Nuclear Science, Stony Brook, NY 11790, USA
}

Received: 9 April 2019; Accepted: 24 April 2019; Published: 28 April 2019

\begin{abstract}
Quarks and gluons are the fundamental constituents of nucleons. Their interactions rather than their mass are responsible for $99 \%$ of the mass of all visible matter in the universe. Measuring the fundamental properties of matter has had a large impact on our understanding of the nucleon structure and it has given us decades of research and technological innovation. Despite the large number of discoveries made, many fundamental questions remain open and in need of a new and more precise generation of measurements. The future Electron Ion Collider (EIC) will be a machine dedicated to hadron structure research. It will study the content of protons and neutrons in a largely unexplored regime in which gluons are expected to dominate and eventually saturate. While the EIC will be the machine of choice to quantify this regime, recent surprising results from the heavy ion community have begun to exhibit similar signatures as those expected from a regime dominated by gluons. Many of the heavy ion results that will be discussed in this document highlight the kinematic limitations of hadron-hadron and hadron-nucleus collisions. The reliability of using as a reference proton-proton (pp) and proton-ion (pA) collisions to quantify and disentangle vacuum and Cold Nuclear Matter (CNM) effects from those proceeding from a Quark Gluon Plasma (QGP) may be under question. A selection of relevant $\mathrm{pp}$ and $\mathrm{pA}$ results which highlight the need of an EIC will be presented.
\end{abstract}

Keywords: QGP; EIC; gluon saturation; nPDF; DIS; CNM

\section{Introduction}

Quarks and gluons, collectively called partons, are the fundamental constituents of protons, neutrons, the atomic nucleus as well as other hadrons. Their interaction is governed by Quantum Chromodynamics (QCD). Understanding QCD, and in particular the confinement of quarks and gluons inside hadrons, is one of today's greatest physics challenges. QCD is the theory of strong interactions and it is expected to describe building blocks of visible matter and their binding in nuclei. While QCD is a well established theory, it contains elements that cannot be calculated and rely mostly on experimental input. ${ }^{1}$ As of today, many fundamental aspects of the theory have not yet been quantified. These aspects include the quantified contribution of partons (and their interactions) to the proton spin, or the mechanisms that permits us to transition from point-like to non-point-like physics.

1 While lattice calculations address these problems directly, results emerging from the lattice typically require large time scales. The accuracy of the obtained results is largely correlated with the amount of computing power allocated to pursuing these calculations. 
Since the discovery of quarks and gluons and the confirmation that they carried color and spin, QCD and related sub-fields have continuously given us discoveries. One of these discoveries is the Quark Gluon Plasma (QGP) formation. This discovery uncovered a new state of matter in which partons were no longer confined to the boundaries of a hadron, but rather acted as free particles.

Evidence of this new state of matter was observed in heavy-ion collisions at the Relativistic Heavy Ion Collider (RHIC) with the discovery of a suppression of high transverse momentum hadrons, also called "jet quenching" [1]. Jet quenching is attributed to a decrease of the energy of the hard partons created during the first stages of a high-energy heavy-ion collision. The formation of the QGP is now understood as being responsible for this loss of energy via interactions with its constituting hot and dense medium. Since its discovery, we have learned many of the interesting properties governing the QGP:

- $\quad$ The QGP behaves as a near-ideal Fermi liquid (almost no frictional resistance or viscosity) [2].

- The mean free path of partons in the QGP is comparable to inter-particle spacing [3].

- Experimental evidence points towards collective motion of particles during the QGP expansion [4].

While more precision measurements are needed, some revealing information has been obtained regarding the QGP onset [5] as it is illustrated in Figure 1 from the The Solenoidal Tracker At RHIC (STAR) experiment. This figure illustrates a classic QGP measurement: particle suppression in heavy ion collisions observed via the central-to-peripheral nuclear modification factor ratio $R_{c p}$, as a function of transverse momentum and center of mass collision energy per nucleon-nucleon collision $\left(\sqrt{s_{N N}}\right)$. A smooth transition is seen as a function of $\sqrt{s_{N N}}$ between enhancement and particle suppression, the latter a signature of the presence of a QGP.

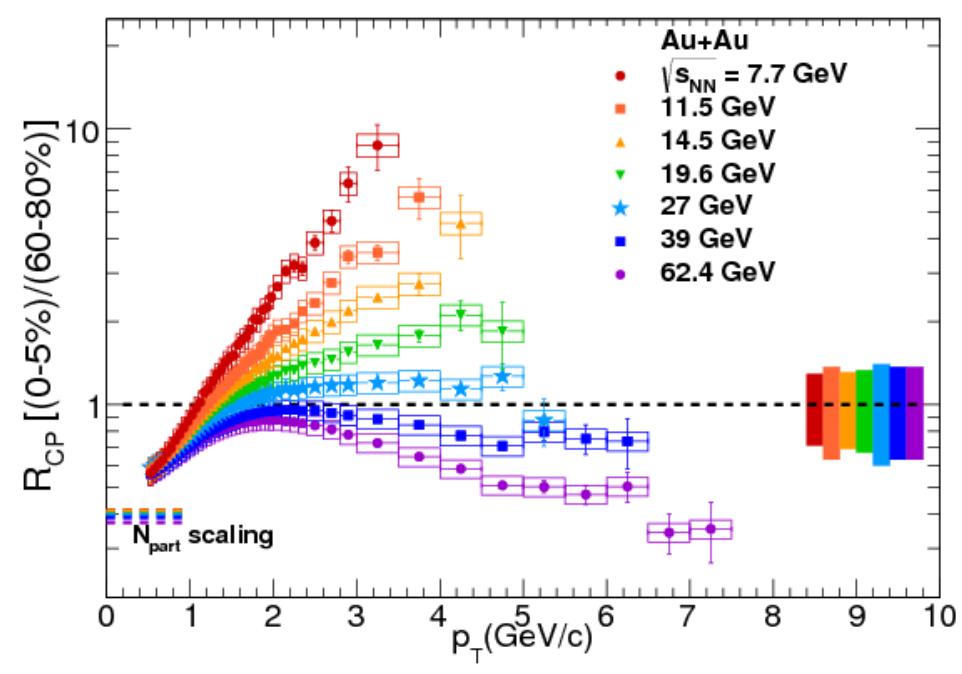

Figure 1. Nuclear modification factor $\left(R_{C P}\right)$ of high- $p_{T}$ hadrons produced in central collisions relative to those produced in peripheral collisions. A QGP onset is observed at collision energies $\sqrt{s_{N N}}>30 \mathrm{GeV}$ while an enhancement is observed at lower energies [5].

Despite the plethora of information we have obtained regarding the QGP, many questions remain open-as an example: (1) How precisely does the plasma acquire its Fermi like fluid (i.e., almost no frictional resistance or viscosity) characteristics? (2) What are the processes in which color-charged quarks and gluons and colorless jets interact with a nuclear medium? (3) Is there a smooth transition for the physics involved in small systems to that of large systems? (4) Finally, when does one transition from a regime of partons to a regime in which gluons dominate?

Indeed, recent puzzling results from proton-proton $(\mathrm{pp})$ and proton-ion $(\mathrm{pA})$ collisions seem to insist we address the above. 


\section{A New Physics Regime}

The interaction between partons is usually described as a function of at least two quantities: the momentum fraction $x$ of the parent nucleon carried by the partons under consideration and the energy/length scale $Q^{2}$ at which the interaction between partons is probed. These two quantities allow one to identify several regimes for $Q C D$, constituting what one calls the QCD landscape and illustrated on Figure 2 (left). For a given $Q^{2}$, as we decrease towards smaller values of $x$, the number of partons is increased-while, for a given $x$ and as we decrease towards smaller values of $Q^{2}$ (reduce the resolution), the size of partons increase. Now, if we vary our kinematics towards small $x$ and small $Q$, one enters a regime characterized by a large number of partons (gluons rather), with overlapping wave functions. This is the phenomena that is known as gluon saturation [6].
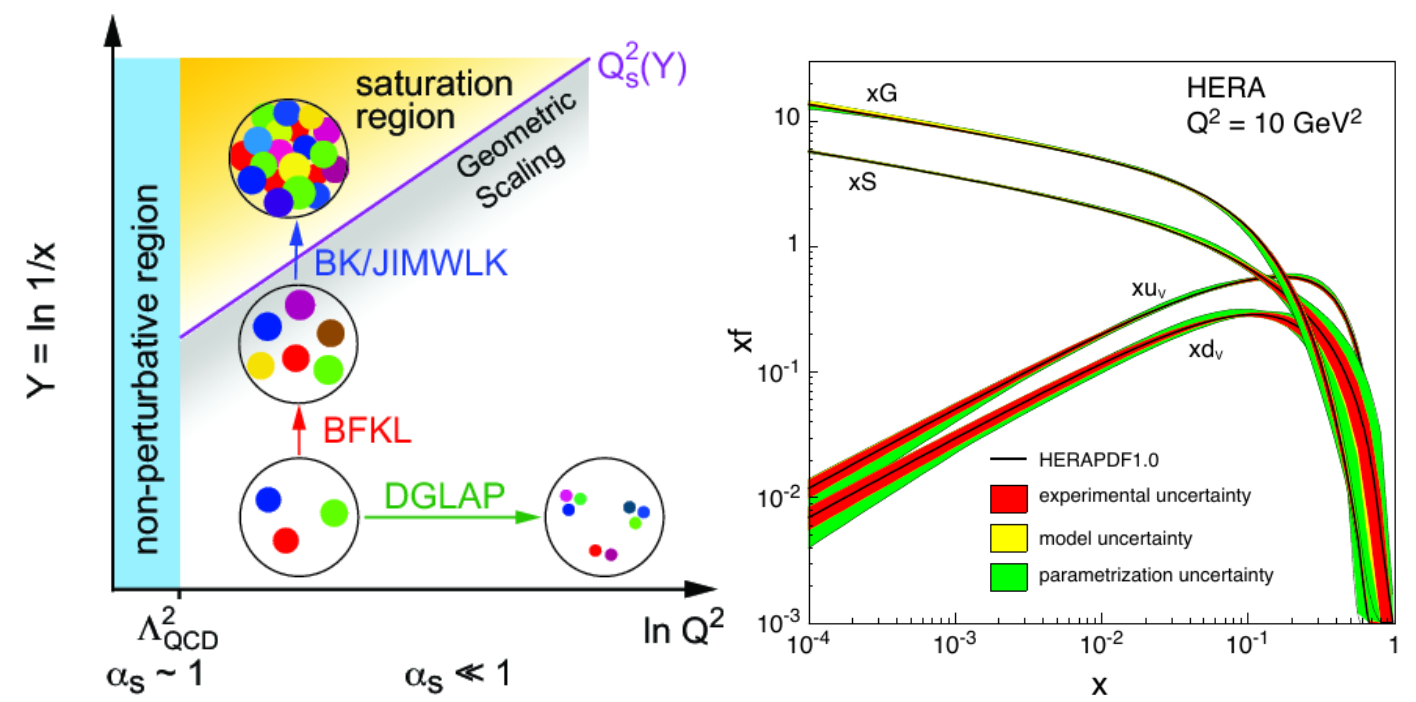

Figure 2. Left: the QCD landscape, the horizontal axis $Q^{2}$ represents the resolution of the probe while the $y$-axis $(\ln (1 / x))$ is related to the parton density. Right: Parton distribution functions in the proton plotted as functions of Bjorken $x$; figures from [7].

For large values of $Q^{2}$, the coupling constant $\alpha_{s}$ is small and one expects scattering directly from point-like bare color charges. perturbative Quantum Chromo Dynamics (pQCD) can be then used to reliably predict the hard scattering of partons. For small values of $Q^{2}$, in a regime relevant for the description of nucleons and nuclei, one probes longer length scales making QCD non-perturbative and very little is thus calculable. For these small values of $Q^{2}$ the content of the nucleon in terms of partons is parameterized using parton distribution functions (PDF) and more recently Generalized Parton Distribution functions (GPD) [8]. Parametrization of PDFs typically requires experimental input (or direct calculations on the lattice). For a given value of $Q^{2}$ and decreasing values of $x$, the density of gluons in the nucleon increases very rapidly (see Figure 2 right). However, for small enough values of $x$, and large enough values of $Q^{2}$ for $\alpha_{s}$ to be considered small, it is expected that this increase eventually saturates, giving rise to a new regime characterized by weakly-coupled but highly correlated gluon matter called Color Glass Condensate (CGC).

A variety of recent Large Hadron Collider (LHC) results indicate that small systems such as pp and pA exhibit signatures typically expected in larger heavy-ion systems (AA collisions) and resulting from the presence of a QGP. A variety of theories exist which aim at providing explanation to these results some of which include (1) presence of a QGP already in these small systems and (2) universal properties of all nuclei (small and large) in a gluon saturation regime. The first of these explanations requires a careful disentangling of the initial state effects. This is not a trivial task since this is usually achieved using these same small systems as a reference. The second explanation can be tested-with coarse precision and large uncertainties-at current colliders. It is clear that a new generation of results, 
such as those that will be performed at the Electron Ion Collider (EIC), will be extremely important to help quantify initial state effects with better precision than what is currently achievable. Furthermore, EIC measurements will be pivotal to precisely pin-down the presence of new physics regimes i.e., gluon saturation.

\section{The Electron Ion Collider}

One of the goals of the lepton-ion (eA) program at an EIC is to unveil the collective behavior of densely packed gluons under conditions where their self-interactions dominate. With its high luminosity and detector acceptance, as well as its span of available collision energies and ion species, the EIC will probe the confined motion as well as the spatial distributions of quarks and gluons inside a nucleus at one tenth of a femtometer resolution. The EIC will be able to detect soft gluons whose energy in the rest frame of the nucleus is less than one tenth of the average binding energy needed to hold the nucleons together to form the nucleus [7]. Thanks to eA collisions with large nuclei, the EIC will reach the saturation regime faster than with ep collisions at similar center of mass energies $(\mathrm{cms})$ energies (Figure 3). This is due to the $x$ and mass number (A) dependence of the saturation scale $Q_{s}$, which goes like:

$$
Q_{s}^{2}(x) \sim A^{1 / 3}(1 / x)^{\lambda}
$$

The EIC will investigate the onset of saturation, explore its properties and reveal its dynamical behavior. It will also provide a kinematically well defined reference to quantify cold nuclear matter effects. For completeness, it is noted that a similar accelerator proposal (LHeC) with complementary kinematic coverage and physics programme is being evaluated by the European Strategy for Particle Physics $[9,10]$.
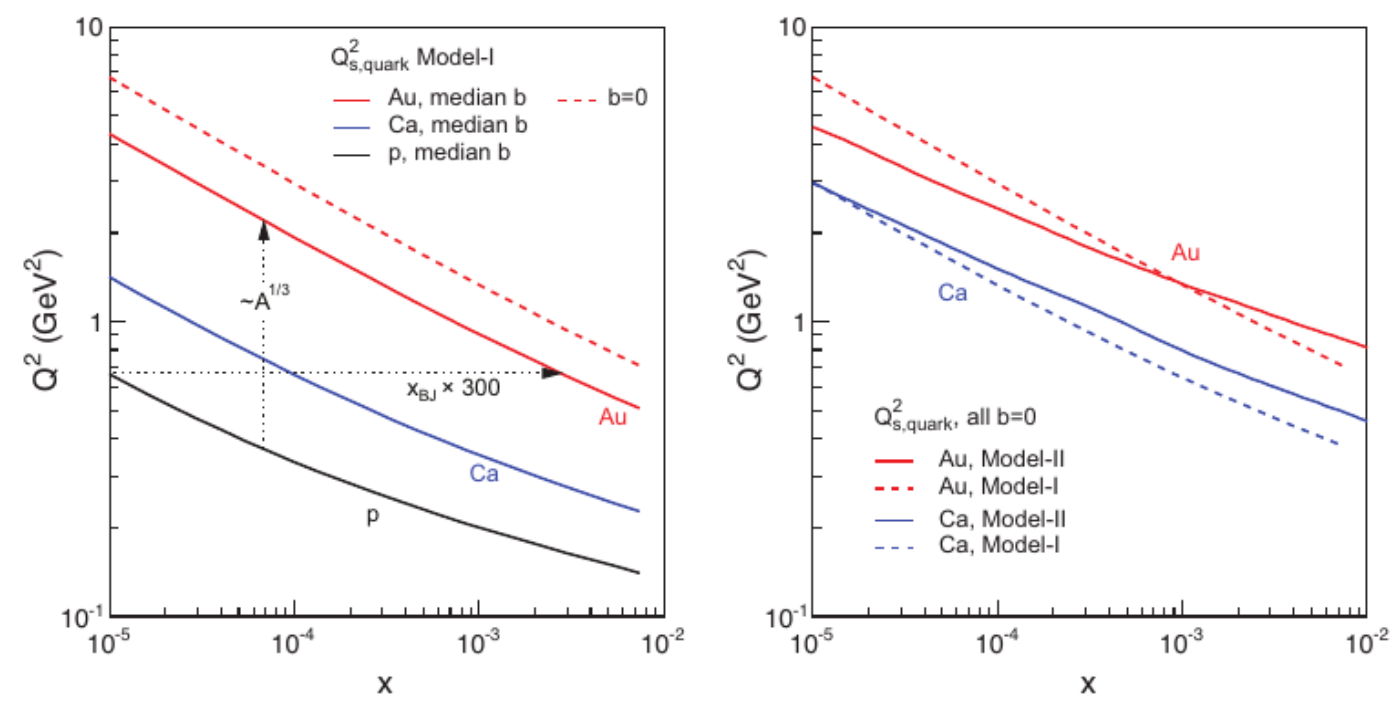

Figure 3. Theoretical expectations for the saturation scale as a function of Bjorken $x$ for the proton along with $\mathrm{Ca}$ and Au nuclei. $Q_{s}^{2} \sim 7 \mathrm{GeV}^{2}$ is reached at $x=10^{-5}$ in e-p collisions at a $\sqrt{s} \sim 1 \mathrm{TeV}$ while in e-Au collisions, only $\sqrt{s} \sim 60 \mathrm{GeV}$ is needed to achieve comparable gluon density and the same saturation scale; figure from [7].

The EIC is considered a key component for the future nuclear physics program in the US and as such is among the key recommendations of the Nuclear Science Advisory Committee (NSAC) Long Range Plan from 2015. It has further received a positive and encouraging report from the National Academy of Sciences [11]. 


\subsection{EIC Requirements}

- Large luminosity $\left(10^{33}-10^{34} \mathrm{~cm}^{-2} \mathrm{~s}^{-1}\right)$,

- Center of mass energy (30-140) GeV,

- Hadron and electron beams with high longitudinal spin polarization,

- Ion beams from deuteron to the heaviest stable nuclei,

- Large detector acceptance, in particular for small angle scattered hadrons,

- Optimized high luminosity and high acceptance running modes.

\subsection{EIC Designs}

The eRHIC design is based on an upgrade to the Relativistic Heavy Ion Collider (RHIC) located at Brookhaven National Laboratory (BNL) in New York:

- New electron injector,

- $5-18 \mathrm{GeV}$ electron energy,

- Energy of heavy ions up to $100 \mathrm{GeV} / \mathrm{u}$,

- $\sqrt{s}: 20-140 \mathrm{GeV}$,

- Peak luminosity of $\sim 0.4 \times 10^{34} \mathrm{~cm}^{-2} \mathrm{~s}^{-1} / \mathrm{A}$ as a base design and $1.0 \times 10^{34} \mathrm{~cm}^{-2} \mathrm{~s}^{-1} / \mathrm{A}$ achievable with strong cooling.

The Jefferson Lab EIC (JLEIC) design is based on an upgrade to the Continuous Electron Beam Accelerator Facility (CEBAF) located at the Jefferson Laboratory in Virginia:

- New hadron injector,

- A "figure-8" layout for the booster and collider rings which preserves spin polarization,

- 3-12 GeV electron energy,

- Energy of heavy ions up to $80 \mathrm{GeV} / \mathrm{u}$ that could be upgraded to $160 \mathrm{GeV} / \mathrm{u}$,

- $\sqrt{s}: 20-100 \mathrm{GeV}$ that could be upgraded to $140 \mathrm{GeV}$,

- Average luminosity per run $\sim 10^{34} \mathrm{~cm}^{-2} \mathrm{~s}^{-1} / \mathrm{A}$.

Both designs have science cases by themselves which require a robust integration with detector designs. An ongoing "Generic Detector for an EIC" research and development peer reviewed program is funded by the United States Department of Energy. Thanks to these funds, an active effort exists in which a variety of detector designs and technologies which meet EIC requirements are being explored and tested. Two such examples are cited: the BeAST and JELIC detector R\&D efforts. See [12] for a complete list of these programs.

\section{Physics at the Energy Frontier: Selection of Recent Results}

A short selection of unexpected heavy ion results measured at the LHC is presented which has prompted interpretations. The presence of a mini QGP in small hadronic systems $[13,14]$ has been proposed as an explanation. Other physics mechanisms that do not involve QGP formation have also been proposed including the existence of a gluon saturation regime $[15,16]$, the string percolation model [17] and others. For an interesting review on the subject, see [18]. It is noted that Monte Carlo generators such as PYTHIA have also been used to describe qualitatively some of the data in this document [19]. 
The selection presented hereafter will highlight the need to better understand small colliding systems if we are to quantify correctly QGP phenomena. The ep and eA collisions of the EIC will undoubtedly contribute to an in-depth understanding of these observations.

\subsection{QGP Onset and Strangeness Enhancement}

Strangeness enhancement was one of the first proposed signatures of the QGP [20]. The QGP expectation was that strange particle yields would be enhanced with respect to their yield in $p p$ collision. The enhancement would then follow a hierachy based on their strange quark content. This implies that a particle with three strange quarks would be enhanced with respect to a particle with two strange quarks, and even more than a particle with only one strange quark. As predicted, strangeness enhancement was observed in AA collisions at the Super Proton Synchrotron (SPS), RHIC and the LHC (Figure 4) [21].
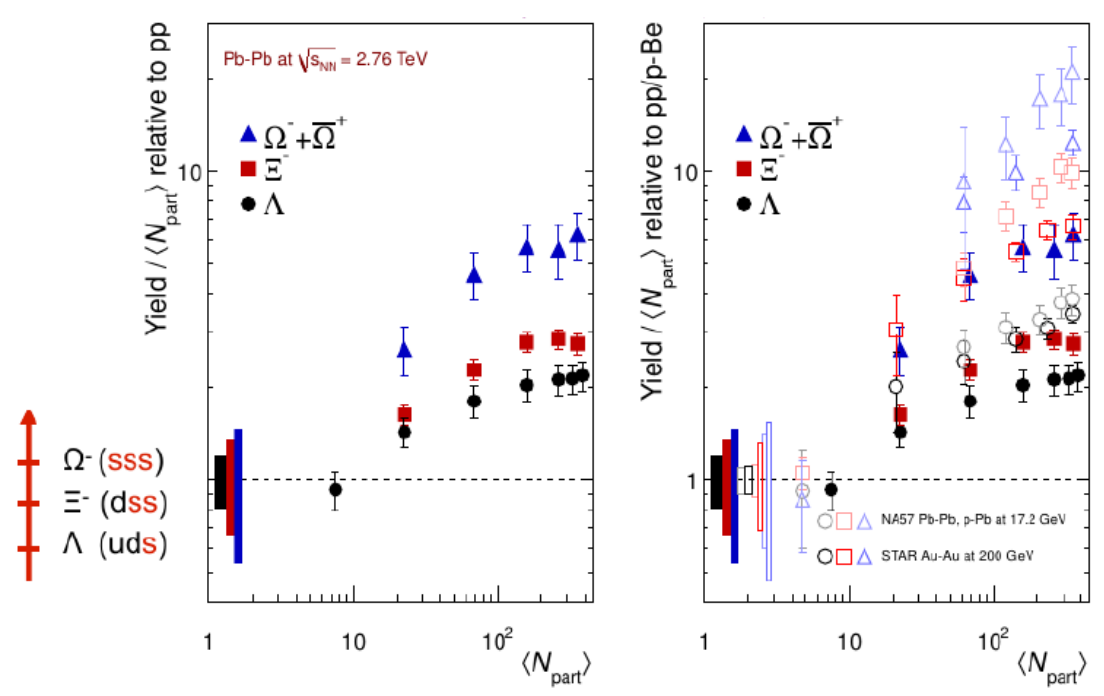

Figure 4. Ratio of strange yields in $\mathrm{PbPb}$ collisions with respect to pp collisions as a function of participants. As it is observed in the figure, an enhancement with respect to pp is observed which is larger for $\Omega$ (sss) than for $\Xi^{-}$(dss) and $\Lambda$ (uds) [21].

What is unexpected, however, is the observation (Figure 5) that an enhancement of strange particles $(K, \Lambda, \Omega)$ with respect to non strange yields (i.e., $\pi)$ is also visible in the most violent high multiplicity $\mathrm{pp}$ and $\mathrm{p}-\mathrm{Pb}$ collisions ${ }^{2}$.

The mechanisms responsible for the observed enhancement in these small systems might indicate that such system may not be relied upon to discern cold from hot nuclear effects. While more experimental insight is needed to interpret the observed enhancement, it has been proposed that the presence of a strong gluon field leading to the nonlinear regime of gluon saturation [7] may explain these observations.

2 Multiplicity is the number of charged particles in the final state. In $\mathrm{pPb}$ and $\mathrm{PbPb}$, this quantity is related to the centrality of the collision. 


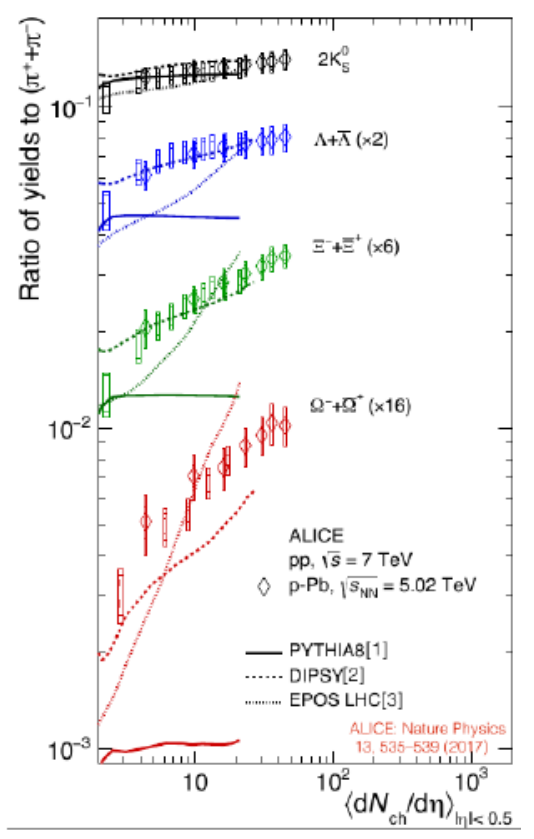

Figure 5. Ratio of strange yields to $\pi^{+}+\pi^{-}$in $\mathrm{pp}, \mathrm{p}-\mathrm{Pb}$ a a function of average particle multiplicity. A smooth transition is observed as a function of particle multiplicity connecting the small (pp) and larger $(\mathrm{pPb})$ systems [22].

\subsection{Heavy Flavor vs. Multiplicity}

Heavy flavor probes are ideal to test QGP properties. The contribution of the QCD vacuum condensate to the masses for the three light quark flavors $(u, d, s)$ considerably exceeds the mass generated by the Higgs field. Charm and beauty masses, on the other hand, are not expected to be affected by this QCD vacuum (Figure 6 left), making them ideal probes of the QGP. The mass of the heavy quark itself provides the hard scale for PQCD calculations. This is in contrast to light quarks which often have to rely on the $p_{T}$ of the final state hadron. In addition, low $p_{T}$ production of charmonia at forward rapidity (where smaller values of $x$ can be reached) is expected to be sensitive to gluon saturation.
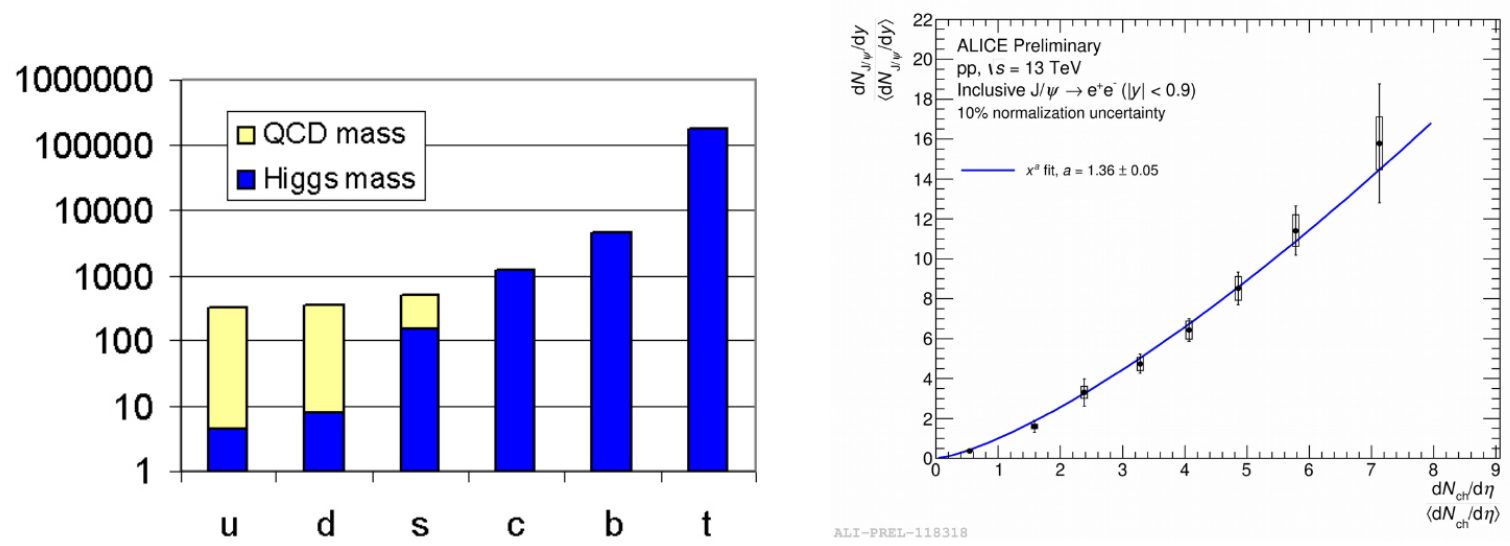

Figure 6. Left: Masses of the six quark flavors. The masses generated by electroweak symmetry breaking (current quark masses) are shown in blue; the additional masses of the light quark flavors generated by spontaneous chiral symmetry breaking in QCD (constituent quark masses) are shown in yellow [23]. Right: Relative $J / \psi$ production (ordinate) yields as a function of the relative number of charged particles per unit of rapidity (abcissa). The blue line corresponds to a fit of a power law function to the data [24]. 
Recent results from the ALICE experiment at the LHC show an event activity dependence of inclusive $J / \psi$ and D mesons [25]. The relative charmonium production yield as a function of the per-event relative charged particle multiplicity shows an increase that is faster than linear in pp collisions (Figure 6 right).

Figure 7 (right) shows a similar measurement performed in $\mathrm{pPb}$ collisions at negative ( $\mathrm{Pb}$-going side), mid and forward rapidity (p-going side). The positive rapidity measurement corresponds to small $x$ values $\left(\sim 10^{-5}\right)$, a range in which gluon saturation may be present. The observation of similar charged particle multiplicity dependence (Figure 7 left) for both open and hidden charm indicates that hadronization may be of lesser importance.

One plausible physics explanation for the previous results is the existence of a gluon saturation regime as it has been discussed in the introduction of this document. In Figure 8, a CCG [15] calculation which includes gluon saturation effects is compared to ALICE measurements in pp collisions. The calculation describes the data.
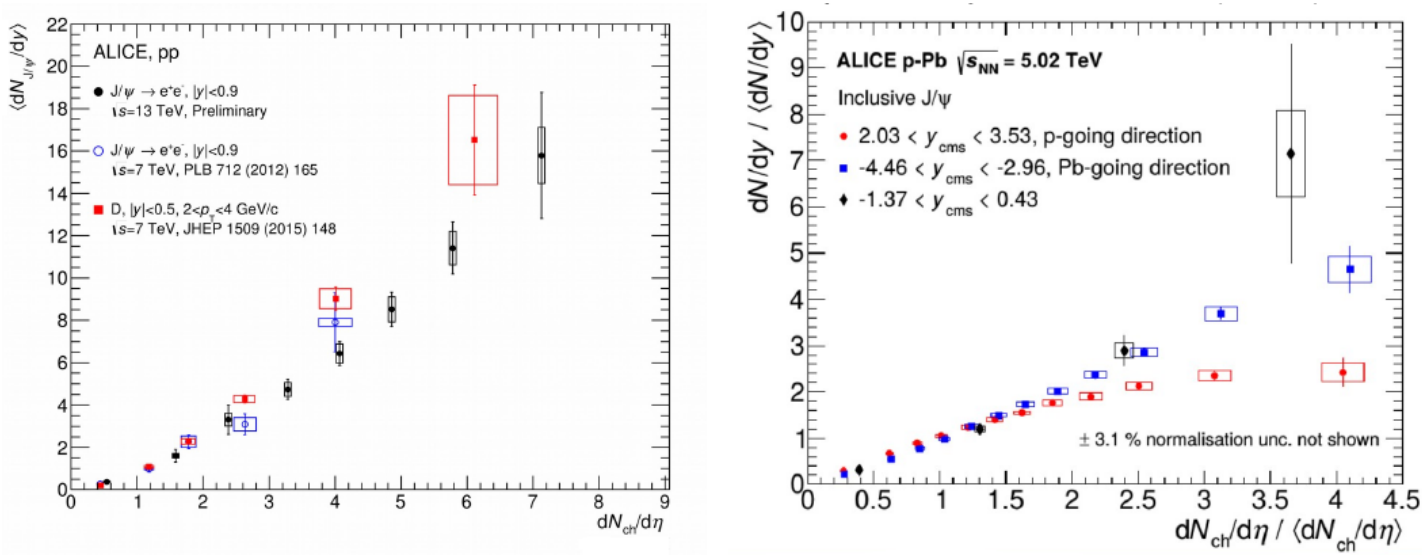

Figure 7. Left: average inclusive $J / \psi$ (closed black and open blue markers), D meson (red closed markers) dependence on charged particle multiplicity in pp collisions and central rapidity. Right: inclusive forward and backward rapidity $J / \psi$ 's dependence on charged particle multiplicity in $\mathrm{p}-\mathrm{Pb}$ collisions [25].

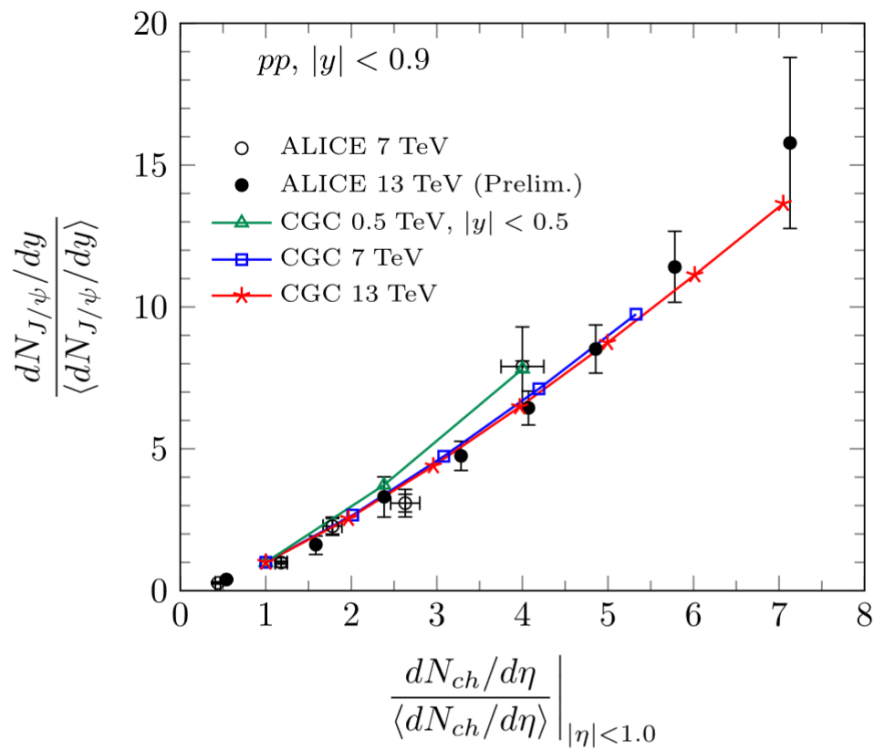

Figure 8. CGC comparisons [15] to recent $J / \psi$ multiplicity results in pp collisions. 


\subsection{Hydrodynamic Flow}

One of the properties of the QGP is that it behaves like a perfect fluid with nearly zero viscosity. This near zero viscosity has been quantified by the correlated momentum anisotropies among the particles produced in the heavy collisions, which result from a common velocity field pattern. This pattern is now identified as collective flow [26]. Among the flow phenomena, two types are highlighted in this document: (1) Radial flow, which typically affects the shape of low $p_{T}$ spectra, and (2) Elliptic Flow $v_{2}$, which is the second coefficient of the Fourier decomposition of particle's momentum azimuthal distributions. This decomposition quantifies the anisotropic particle density which emerges from two nuclei interacting in semi central collisions. A non zero $v_{2}$ implies early thermalization of the medium and it is considered a signature of the QGP.

Baryon to meson ratios obtained in $\mathrm{PbPb}$ collisions as shown in Figure 9 illustrate the effect of radial flow. Radial flow will push hadrons from low $p_{T}$ towards intermediate $p_{T}$. The effect is expected to be stronger for baryons than for mesons, resulting in a bump in the baryon-to-meson ratio (here proton-to-pions ), which depends on the centrality of the collision. Until recently, this was well understood in heavy-ion collisions. What is unexpected, however, is the observation of a similar effect in pp and pPb collisions as shown in Figures 10 and 11. The results would naively imply that thermalization is occurring already in these small systems.

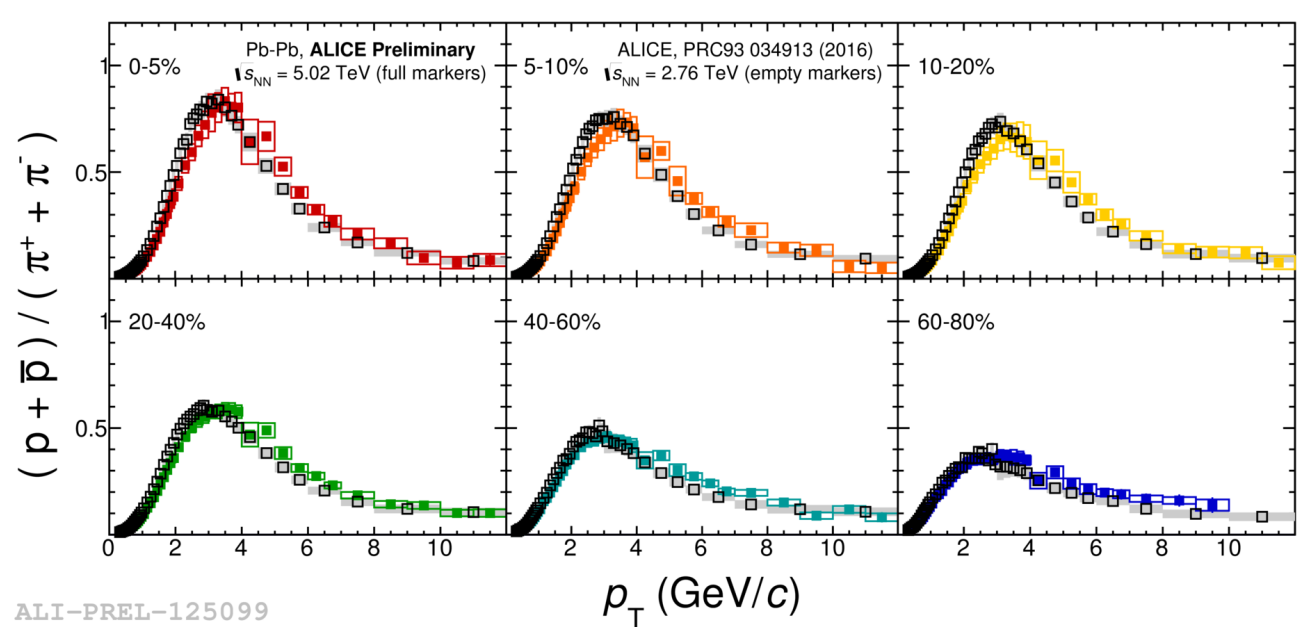

Figure 9. Proton to pion ratio in $\mathrm{PbPb}$ collisions as a function of $p_{T}$ at two $\sqrt{s_{N N}}$ and six centrality classes.

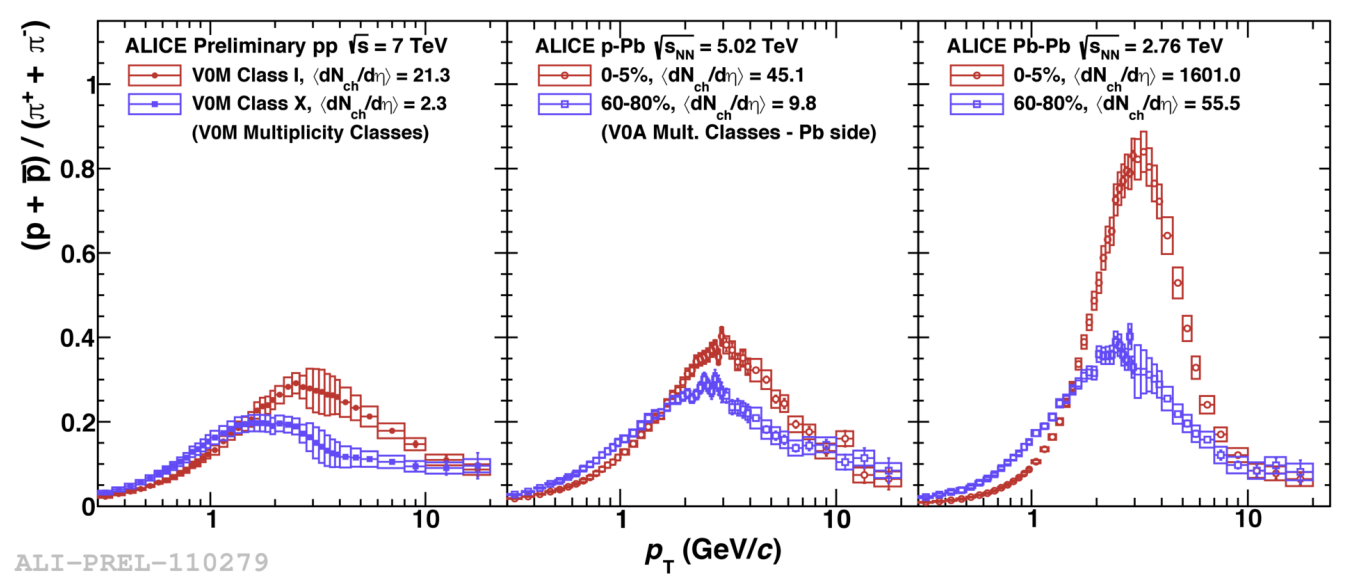

Figure 10. Proton to pion ratio as a function of $p_{T}$ in $\mathrm{pp}$ (left), $\mathrm{pPb}$ (middle) and $\mathrm{PbPb}$ (right) collisions. The measurements are classified as a function of charged particle multiplicity. 


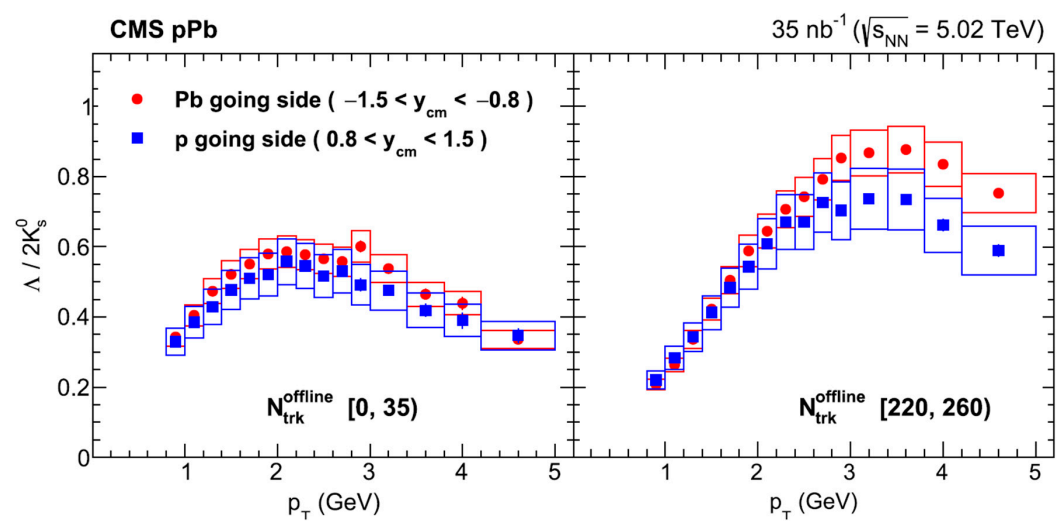

Figure 11. Ratios of $\mathrm{p}_{\mathrm{T}}$ spectra, in forward and backward rapidity regions in $\mathrm{pPb}$ collisions at $\sqrt{s_{N N}}=5.02 \mathrm{TeV}$. Results are presented for two particle multiplicity ranges; figure and details are taken from [27].

Light meson flow $v_{2}$ results in $\mathrm{PbPb}$ [28] and reported by the ALICE collaboration are shown in Figure 12 (top figures). At low $p_{T}$, as it is the case for many other flow results, the trend is understood as being consistent with a collective expansion within the QGP and has been successfully explained by hydrodynamic models [29]. At intermediate and high $p_{T}$ constituent quark number scaling takes over (dressed quarks), all mesons fall together and baryons climb above by $\sim 1 / 3$. What is intriguing on the other hand is that similar signatures are observed in $\mathrm{pPb}$ (Figure 12 bottom and Figure 13) and pp collisions (Figure 13 bottom right). Effects that can cause the current observations are either due to initial state effects (saturation), or final state effects (expansion and/or thermal equilibrium). More recently, quantum entanglement has been suggested as a possible explanation [30-32] as well as double parton scattering coupled with the elliptic gluon Wigner distributions (these account for the impact parameter dependence of hard scatterings involving the unintegrated gluon distributions) [33]. This phenomena could be elucidated with a variety of probes at the EIC's lepton-nucleon program, including diffractive measurements of dijet production.
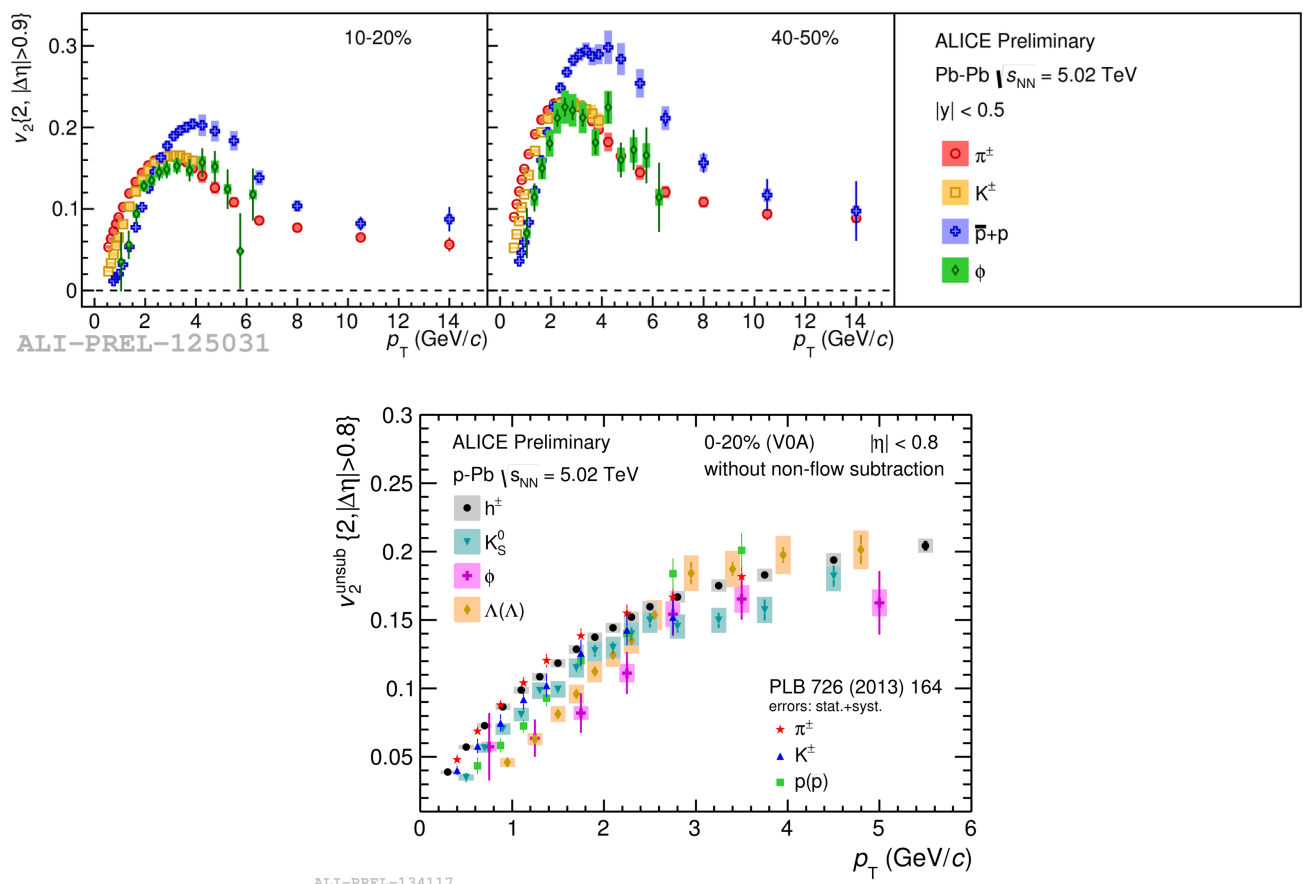

Figure 12. Top figures: elliptic flow $v_{2}$ in $\mathrm{PbPb}$ collisions as a function of $p_{T}$ in two centrality classes and four particle species [28]; Bottom figure: Elliptic flow $v_{2}$ in pPb collisions [34]. 

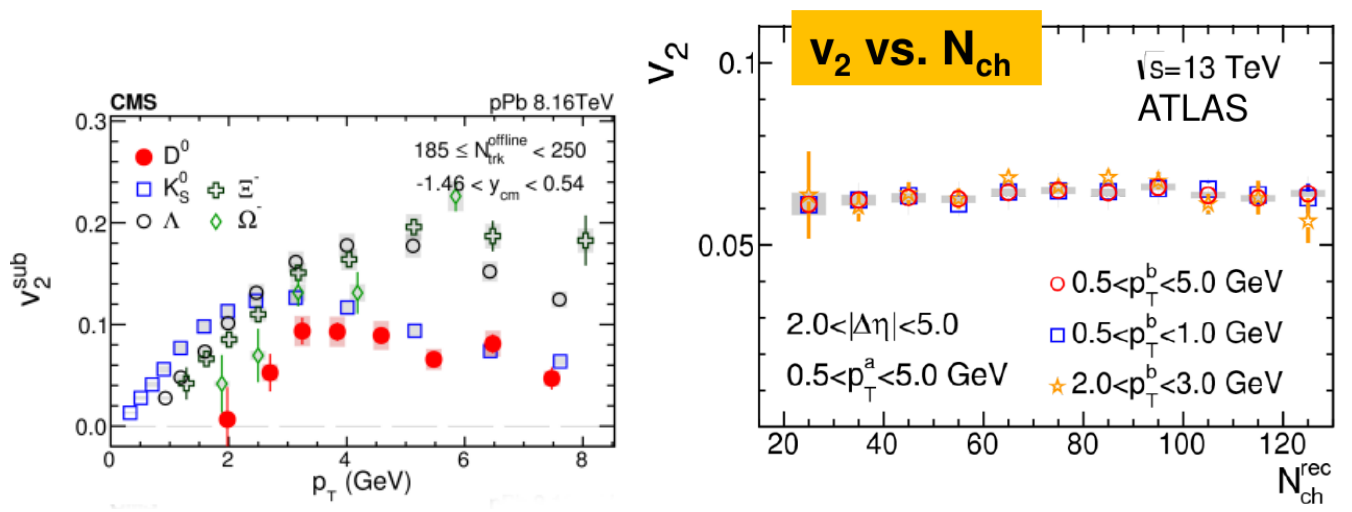

Figure 13. Left: $v_{2}$ flow as a function of $p_{T}$ of charm and strange hadrons in high-multiplicity $\mathrm{pPb}$ collisions at $\sqrt{s_{N N}}=8.16 \mathrm{TeV}$ (CMS Collaboration [35]); Right: $v_{2}$ as a function of $p_{T}$ in pp collisions at $\sqrt{s}=13 \mathrm{TeV}$ (ATLAS Collaboration [36].)

\subsection{Nuclear Modification Factor and Energy Loss in the Medium}

The nuclear modification factor $R_{A A}$ is an observable used to quantify the effect of the nuclear medium on particle production. $R_{A A}$ consists of measuring invariant spectra as a function of $p_{T}$ of particles produced in heavy ion collisions and compared to reference data (pp) at the same energy and scaled by the number of binary collisions. $R_{A A}$ is defined as follows:

$$
R_{A A}=\frac{\mathrm{AA}}{\text { scaled pp }}=\frac{d^{2} N_{\mathrm{AA}} / d p_{T} d y}{<N_{\text {coll }}>d^{2} N_{\mathrm{pp}} / d p_{T} d y}
$$

Values greater than unity would be an indication of production enhancement, while values less than unity will indicate particle suppression in the QGP.

While partons are expected to lose energy when propagating through the dense QGP medium, it is also expected that the amount of energy loss will depend on the parton type and the medium properties. A large number of results, such as those in Figure 14, indicate that the amount of suppression observed in heavy ion collisions is irrelevant of particle mass (or quark content) at high enough $p_{T}$.
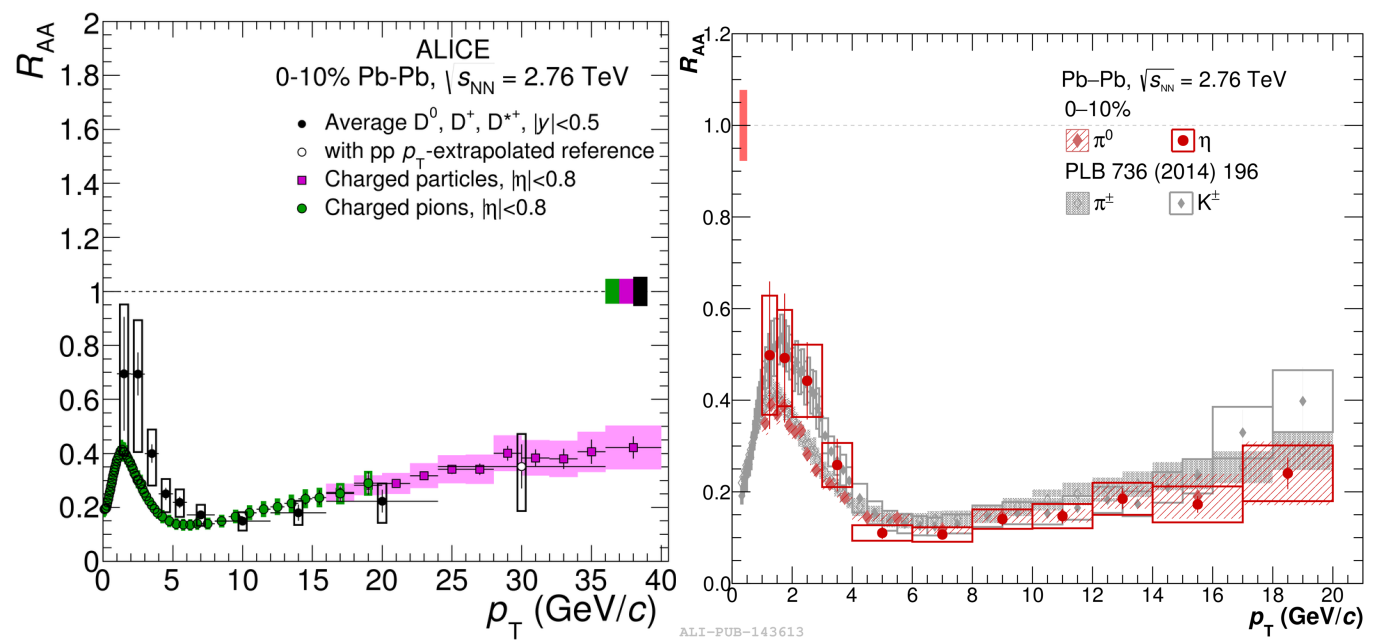

Figure 14. Left: Prompt D-meson $R_{A A}$ as a function of $p_{T}$ compared to the nuclear modification factors of charged pions and charged particles in the $0-10 \%$ centrality class [37]. Right: $R_{A A}$ of neutral and charged pions, kaons and eta meson [38]. 
$R_{A A}$ results could largely benefit from independent measurements at the EIC. Measurements such as those illustrated in Figure 15 will study the response of the nuclear medium to a fast moving quark $[7,39]$ and allow proper understanding of hadronization mechanisms.
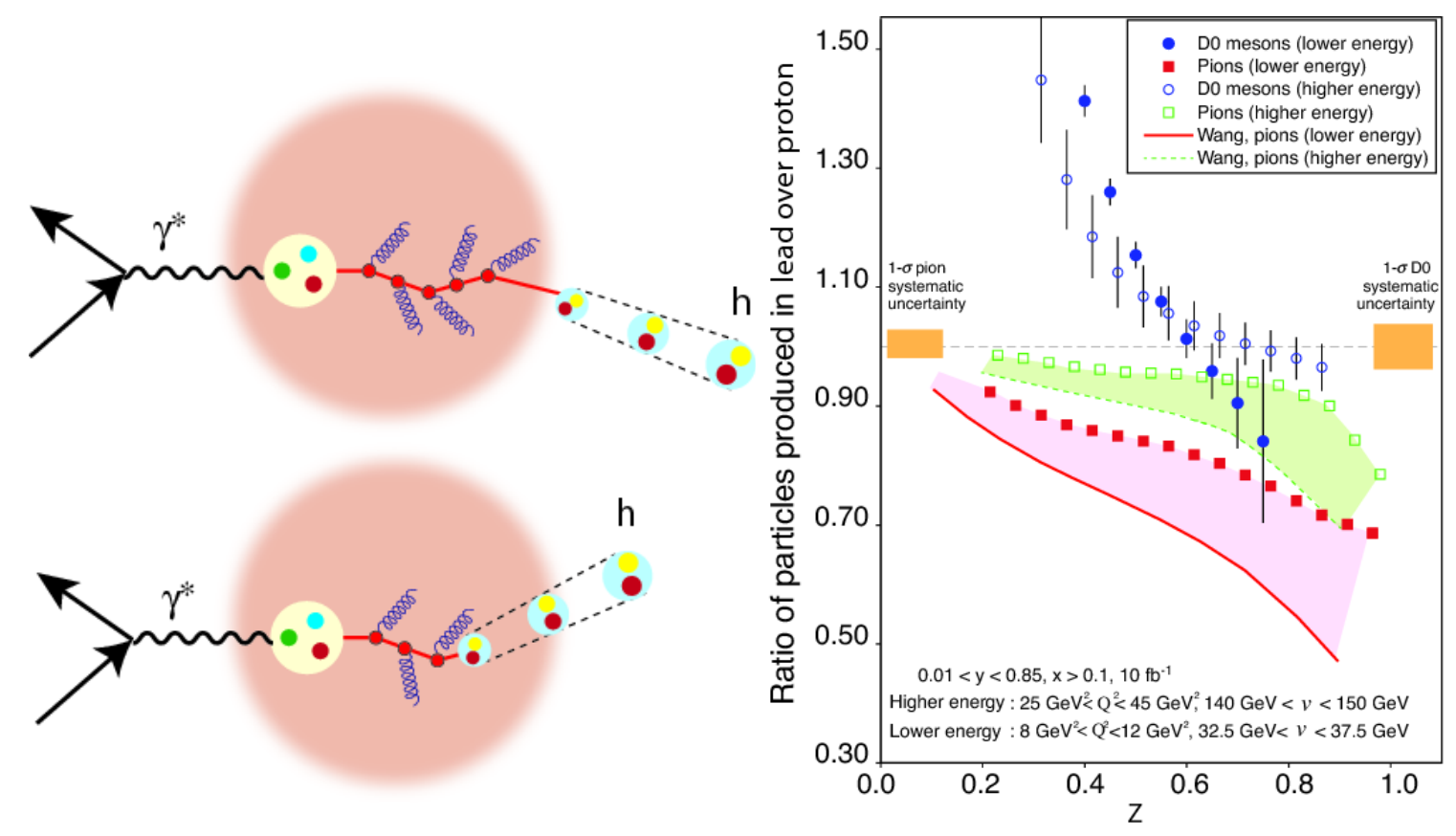

Figure 15. Left: Hadronization schematic illustrating the interaction of a parton moving through cold nuclear matter: the hadron is formed outside (top) or inside (bottom) the nucleus. Right: Ratio of semi-inclusive cross section for producing a pion (red) composed of light quarks, and a $\mathrm{D}^{0}$ meson (blue) composed of heavy quarks in e-Pb collisions to e- $\mathrm{d}$ collisions, plotted as function of $\mathrm{z}$, the ratio of the momentum carried by the produced hadron to that of the virtual photon $\left(\gamma^{*}\right)$, as shown in the plots on the left; figures and descriptions taken from [7].

\subsection{Nuclear Parton Distribution Functions}

Finally, a careful evaluation of initial state effects such as nuclear modifications of Parton Distribution Functions (nPDFs) is also needed in order to correctly quantify hot nuclear effects present. nPDFs refer to the difference observed between nuclear (bound nucleons) PDFs and free nucleons PDFs (proton, neutron). The nuclear modification of PDFs is due to the interactions between partons from different nucleons. As such, precise measurements of nPDFs are essential in order to understand cold nuclear matter effects that may be convoluted with current heavy ion results. Figure 16 illustrates (in grey) the uncertainty of gluon distributions in the lead nucleus, which is rather large at both low and high $x$. Measurements that aim at at improving the precision on nPDF are proposed key measurements of the EIC [7]. 

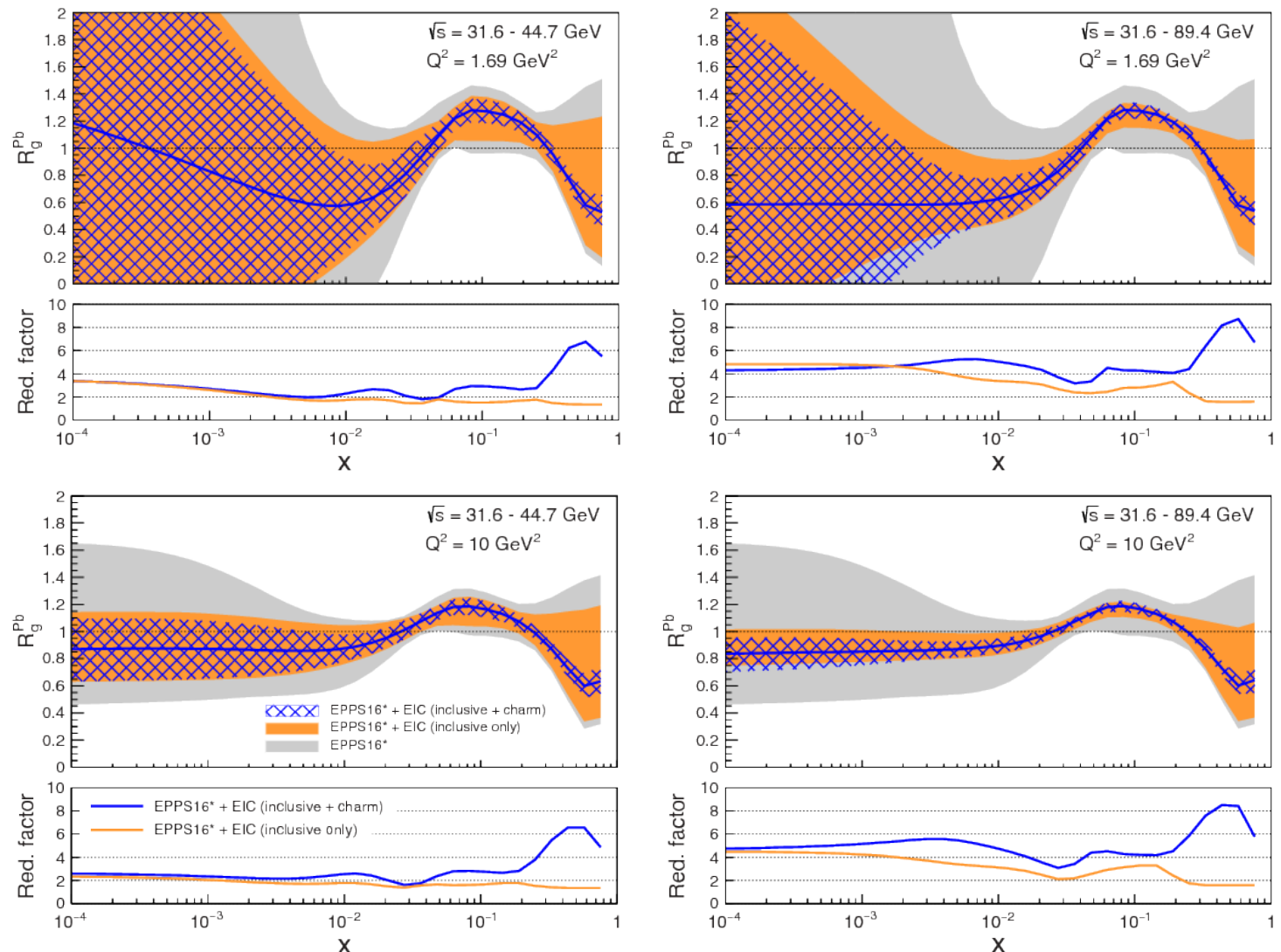

Figure 16. Left: The ratio $R_{g}^{P b}$, from EPPS16*, of gluon distributions in a lead nucleus relative to the proton, for two different momentum transfers $Q^{2}$ possible at the EIC (higher on the bottom figures). Right figures correspond to a larger center of mass energy range considered. The grey band represents the current theoretical uncertainty. The orange (blue hatched) band includes the EIC simulated inclusive (charm quark) reduced cross-section data. The lower panel in each plot shows the reduction factor in the uncertainty with respect to the baseline fit; figures from [40].

\section{Conclusions}

QCD studies have given us decades of discoveries. Many open questions remain on how the transition from a small system to a dense system takes place: this information is needed to fully understand the properties of the QGP. The current document has given a selection of results that may be better understood and quantified with a new generation of lepton-ion experiments at the EIC.

Funding: This research received no external funding.

Acknowledgments: The author thanks the Center for Frontiers on Nuclear Science for the support that enabled promoting the topics outlined in this document.

Conflicts of Interest: The author declares no conflict of interest.

\section{References}

1. Adcox, K. et al. [PHENIX Collaboration]. Suppression of hadrons with large transverse momentum in central Au+Au collisions at $\sqrt{s_{N N}}=130-G e V$. Phys. Rev. Lett. 2002, 88, 022301. [CrossRef] [PubMed]

2. Cao, C.; Elliott, E.; Joseph, J.; Wu, H.; Petricka, J.; Schäfer, T.; Thomas, J.E. Universal Quantum Viscosity in a Unitary Fermi Gas. Science 2011, 331, 58-61. [CrossRef] [PubMed]

3. Shuryak, E. Physics of Strongly coupled Quark-Gluon Plasma. Prog. Part. Nucl. Phys. 2009, 62, 48-101. [CrossRef]

4. Adler, C. et al. [STAR collaboration]. Elliptic flow from two and four particle correlations in $\mathrm{Au}+\mathrm{Au}$ collisions at $\mathrm{s}(\mathrm{NN})^{* *}(1 / 2)=130-\mathrm{GeV}$. Phys. Rev. C 2002, 66, 034904. [CrossRef] 
5. Adamczyk, L.; Adams, J.R.; Adkins, J.K.; Agakishiev, G.; Aggarwal, M.M.; Ahammed, Z.; Ajitanand, N.N.; Alekseev, I.; Anderson, D.M.; Aoyama, R.; et al. Beam Energy Dependence of Jet-Quenching Effects in $\mathrm{Au}+\mathrm{Au}$ Collisions at $\sqrt{s_{\mathrm{NN}}}=7.7,11.5,14.5,19.6,27,39$, and $62.4 \mathrm{GeV}$. Phys. Rev. Lett. 2018, 121, 032301. [CrossRef]

6. Gelis, F.; Iancu, E.; Jalilian-Marian, J.; Venugopalan, R. The Color Glass Condensate. Ann. Rev. Nucl. Part. Sci. 2010, 60, 463-489. [CrossRef]

7. Accardi, A.; Albacete, J.L.; Anselmino, M.; Armesto, N.; Aschenauer, E.C.; Bacchetta, A.; Boer, D.; Brooks, W.K.; Burton, T.; Chang, N.-B.; et al. Electron Ion Collider: The Next QCD Frontier. Eur. Phys. J. A 2016, 52, 268. [CrossRef]

8. Ji, X. Generalized parton distributions. Ann. Rev. Nucl. Part. Sci. 2004, 54, 413-450. [CrossRef]

9. Bruning, O.; Klein, M. Exploring the Energy Frontier with Deep Inelastic Scattering at the LHC A Contribution to the Update of the European Strategy on Particle Physics; CERN: Geneva, Switzerland, 2018.

10. Klein, M. Future Deep Inelastic Scattering with the LHeC. In From My Vast Repertoire ...: Guido Altarelli's Legacy; Levy, A., Forte, S., Ridolfi, G., Eds.; World Scientific Publishers: Singapore, 2019; pp. 303-347.

11. An Assessment of U.S.-Based Electron-Ion Collider Science. Available online: https://www.nap.edu/ catalog/25171/an-assessment-of-us-based-electron-ion-collider-science (accessed on 28 March 2019).

12. EIC Detector Research and Developement. Available online: https://wiki.bnl.gov/conferences/index.php/ EIC_R\%25D (accessed on 28 March 2019).

13. Zhang, X.; Liao, J. Jet Quenching and Its Azimuthal Anisotropy in AA and possibly High Multiplicity pA and dA Collisions. arXiv 2013, arXiv:1311.5463

14. Park, C.; Shen, C.; Jeon, S.; Gale, C. Rapidity-dependent jet energy loss in small systems with finite-size effects and running coupling. Nucl. Part. Phys. Proc. 2017, 289-290, 289-292. [CrossRef]

15. Ma, Y.Q.; Tribedy, P.; Venugopalan, R.; Watanabe, K. Event engineering studies for heavy flavor production and hadronization in high multiplicity hadron-hadron and hadron-nucleus collisions. Phys. Rev. D 2018, 98, 074025. [CrossRef]

16. Mace, M.; Skokov, V.V.; Tribedy, P.; Venugopalan, R. Systematics of azimuthal anisotropy harmonics in proton-nucleus collisions at the LHC from the Color Glass Condensate. Phys. Lett. B 2019, 788, 161-165. [CrossRef]

17. Braun, M.A.; Dias de Deus, J.; Hirsch, A.S.; Pajares, C.; Scharenberg, R.P.; Srivastava, B.K. De-Confinement and Clustering of Color Sources in Nuclear Collisions. Phys. Rep. 2015, 599, 1-50. [CrossRef]

18. Dusling, K.; Li, W.; Schenke, B. Novel collective phenomena in high-energy proton-proton and proton-nucleus collisions. Int. J. Mod. Phys. E 2016, 25, 1630002. [CrossRef]

19. Sjöstrand, T.; Ask, S.; Christiansen, J.R.; Corke, R.; Desai, N.; Ilten, P.; Mrenna, S.; Prestel, S.; Rasmussen, C.O.; Skands, P.Z. An Introduction to PYTHIA 8.2. Comput. Phys. Commun. 2015, 191, 159-177. [CrossRef]

20. Rafelski, J.; Muller, B. Strangeness Production in the Quark-Gluon Plasma. Phys. Rev. Lett. 1982, 48, 1066; Erratum in Phys. Rev. Lett. 1986, 56, 2334. [CrossRef]

21. Abelev, B.B. et al. [ALICE Collaboration]. Multi-strange baryon production at mid-rapidity in $\mathrm{Pb}-\mathrm{Pb}$ collisions at $\sqrt{s_{N N}}=2.76$ TeV. Phys. Lett. 2014, B728, 216-227; Erratum in Phys. Lett. B 2014, 734, 409. [CrossRef]

22. Adam, J. et al. [ALICE Collaboration]. Enhanced production of multi-strange hadrons in high-multiplicity proton-proton collisions. Nat. Phys. 2017, 13, 535-539.

23. Muller, B. Hadronic signals of deconfinement at RHIC. Nucl. Phys. A 2005, 750, 84-97. [CrossRef]

24. Weber, S.G. Measurement of $J / \psi$ production as a function of event multiplicity in pp collisions at $\sqrt{s}=13 \mathrm{TeV}$ with ALICE. Nucl. Phys. A 2017, 967, 333-336. [CrossRef]

25. Adamová, D. et al. [ALICE Collaboration]. $J / \psi$ production as a function of charged-particle pseudorapidity density in p-Pb collisions at $\sqrt{s_{\mathrm{NN}}}=5.02 \mathrm{TeV}$. Phys. Lett. B 2018, 776, 91-104.

26. Heinz, U.; Snellings, R. Collective flow and viscosity in relativistic heavy-ion collisions. Ann. Rev. Nucl. Part. Sci. 2013, 63, 123-151. [CrossRef]

27. Khachatryan, V. et al. [The CMS Collaboration]. Multiplicity and rapidity dependence of strange hadron production in pp, pPb, and PbPb collisions at the LHC. Phys. Lett. B 2017, 768, 103-129. [CrossRef]

28. Noferini, F. ALICE results from Run-1 and Run-2 and perspectives for Run-3 and Run-4. J. Phys. Conf. Ser. 2018, 1014, 012010. [CrossRef]

29. Hirano, T.; van der Kolk, N.; Bilandzic, A. Hydrodynamics and Flow. Lect. Notes Phys. 2010, 785, $139-178$. 
30. Feal, X.; Pajares, C.; Vazquez, R.A. Thermal behavior and entanglement in $\mathrm{Pb}-\mathrm{Pb}$ and $p-p$ collisions. Phys. Rev. C 2019, 99, 015205. [CrossRef]

31. Bellwied, R. Quantum entanglement in the initial and final state of relativistic heavy ion collisions. J. Phys. Conf. Ser. 2018, 1070, 012001. [CrossRef]

32. Baker, O.K.; Kharzeev, D.E. Thermal radiation and entanglement in proton-proton collisions at energies available at the CERN Large Hadron Collider. Phys. Rev. D 2018, 98, 054007. [CrossRef]

33. Hagiwara, Y.; Hatta, Y.; Xiao, B.W.; Yuan, F. Elliptic flow in small systems due to elliptic gluon distributions? Phys. Lett. B 2017, 771, 374-378. [CrossRef]

34. Abelev, B.B. et al. [ALICE Collaboration]. Long-range angular correlations of $\mathrm{B}, \mathrm{K}$ and $\mathrm{p}$ in $\mathrm{p}$-Pb collisions at $\sqrt{s_{\mathrm{NN}}}=5.02 \mathrm{TeV}$. Phys. Lett. B 2013, 726, 164-177. [CrossRef]

35. Sirunyan, A.M. et al. [CMS Collaboration]. Elliptic flow of charm and strange hadrons in high-multiplicity $\mathrm{pPb}$ collisions at $\sqrt{s_{\mathrm{NN}}}=8.16 \mathrm{TeV}$. Phys. Rev. Lett. 2018, 121, 082301. [CrossRef]

36. Aad, G. et al. [ATLAS Collaboration]. Observation of Long-Range Elliptic Azimuthal Anisotropies in $\sqrt{s}=13$ and $2.76 \mathrm{TeV} p p$ Collisions with the ATLAS Detector. Phys. Rev. Lett. 2016, 116, 172301. [CrossRef]

37. Adam, J. et al. [ALICE Collaboration]. Transverse momentum dependence of D-meson production in $\mathrm{Pb}-\mathrm{Pb}$ collisions at $\sqrt{\mathrm{S}_{\mathrm{NN}}}=2.76 \mathrm{TeV}$. J. High Energy Phys. 2016, 2016, 081. [CrossRef]

38. Acharya, S. et al. [ALICE Collaboration]. Neutral pion and $\eta$ meson production at mid-rapidity in $\mathrm{Pb}-\mathrm{Pb}$ collisions at $\sqrt{s_{N N}}=2.76 \mathrm{TeV}$. Phys. Rev. C 2018, 98, 044901. [CrossRef]

39. Aschenauer, E.C.; Fazio, S.; Lee, J.H.; Mantysaari, H.; Page, B.S.; Schenke, B.; Ullrich, T.; Venugopalan, R.; Zurita, P. The electron-ion collider: Assessing the energy dependence of key measurements. Rep. Prog. Phys. 2019, 82, 024301. [CrossRef] [PubMed]

40. Fazio, S. Nuclear Parton Distributions at the future Electron-Ion Collider. PoS 2018, DIS2017, 084,

(C) 2019 by the authors. Licensee MDPI, Basel, Switzerland. This article is an open access article distributed under the terms and conditions of the Creative Commons Attribution (CC BY) license (http:/ / creativecommons.org/licenses/by/4.0/). 